\title{
Análise Numérica da Equação da Onda Imagem na Profundidade
}

J. SCHLEICHER, A. NOVAIS, F.P. MUNERATO, Departamento de Matemática Aplicada, IMECC, UNICAMP, 13081-970 Campinas, SP, Brasil.

Resumo. A equação da onda imagem para o problema de remigração na profundidade é uma equação diferencial parcial de segunda ordem semelhante à equação da onda acústica. Neste trabalho, determinamos as condições de consistência e estabilidade de um esquema de diferenças finitas para esta equação. Observa-se em testes numéricos que, além dos critérios de estabilidade, a forma da implementação do esquema também é essencial para que o erro numérico seja aceitável.

\section{Introdução}

A equação da onda imagem para o problema de remigração na profundidade é uma equação diferencial parcial semelhante à equação da onda acústica. O objetivo deste trabalho consiste na análise numérica desta equação com o fim de encontrar uma implementação estável que forneça uma solução convergente.

O problema da remigração provém do processamento sísmico e o seu objetivo é a construção de uma nova imagem do subsolo a partir de uma obtida anteriormente pelo processo de migração, utilizando uma outra velocidade de migração. Por migração se conhece o processo que, na geofísica, tem como objetivo a reconstrução de uma imagem das camadas geológicas no subsolo a partir da imagem distorcida no tempo, obtida mediante um levantamento sísmico, i.e., mediante geração de ondas no subsolo e registro do movimento resultante das partículas da superfície da terra. Para a realização da migração, é necessário conhecer um modelo das velocidades de propagação das ondas no subsolo em consideração, a chamada velocidade demigração (para mais detalhes ver por exemplo [6]).

Quando é necessária a atualização da imagem do subsolo, obtida anteriormente mediante o uso de um modelo de velocidade de migração incorreto, correspondente a um modelo melhor, fala-se de "remigração" $([3,4])$. Geralmente, quando é feita uma migração, o modelo de velocidade correto não é conhecido e, portanto, o fato de podermos mudar a velocidade de migração e construirmos uma nova imagem migrada correspondente é de extrema importância. Observa-se que as novas imagens de um refletor (i.e., uma fronteira entre camadas geológicas) para diferentes valores da velocidade de migração comportam-se de maneira análoga à propagação de frentes de onda. A variável de propagação, que no caso da propagação de ondas físicas é representada pelo tempo, é, no caso das ondas imagens, a velocidade de 


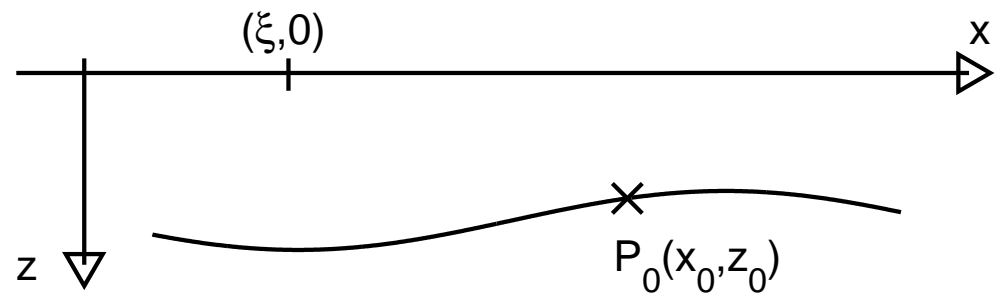

Figura 1: Representação pontual do refletor $P_{0}\left(x_{0}, z_{0}\right)$.

migração. Esta "propagação de imagens" é descrita pela chamada equação da onda imagem, dada por $[2,3]$,

$$
p_{x x}+p_{z z}+\frac{v}{z} p_{v z}=0
$$

A sua condição inicial é dada por $p\left(x, z, v_{0}\right)=p_{0}(x, z)$, que é uma seção migrada com a velocidade errada $v_{0}$.

Para obter uma solução aproximada da onda imagem, a qual é uma equação diferencial parcial linear, pode-se usar o método de diferenças finitas $([5,7,8])$. Neste trabalho, estudamos a estabilidade, a consistência e, assim, a convergência de um esquema de diferenças finitas escolhido. O estudo de estabilidade consiste de uma investigação teórica, bem como de testes numéricos.

\section{Equação da onda imagem}

A dedução da equação (1.1) pode ser feita a partir da imagem remigrada de um ponto refletor, porque todo refletor pode ser entendido como uma seqüência de pontos refletores. Assim, sua dedução se separa em dois passos: deduzir a posição da imagem remigrada de um ponto refletor e achar uma equação diferencial parcial que descreva a variação desta posição com a velocidade de migração ([3]). Nesta seção resumimos brevemente esta dedução.

Primeiramente, supomos um ponto refletor $P_{0}\left(x_{0}, z_{0}\right)$ localizado no subsolo (Figura 1). O tempo $t$ de propagação da onda emitida pela fonte, situada na superfície em $(\xi, 0)$, até ser captada pelo receptor, situado no mesmo ponto $(\xi, 0)$, é expresso em função de $\xi$, por

$$
t(\xi)=\frac{2}{v_{0}} \sqrt{\left(x_{0}-\xi\right)^{2}+z_{0}^{2}}
$$

onde $v_{0}$ é a velocidade de propagação no subsolo, supostamente homogêneo, i.e., $v_{0}$ é constante.

Agora podemos pensar que para um dado ponto $(\xi, 0)$, temos um conjunto de pontos que satisfaz a equação (2.2) para a mesma velocidade $v_{0}$, isto é, dado um 

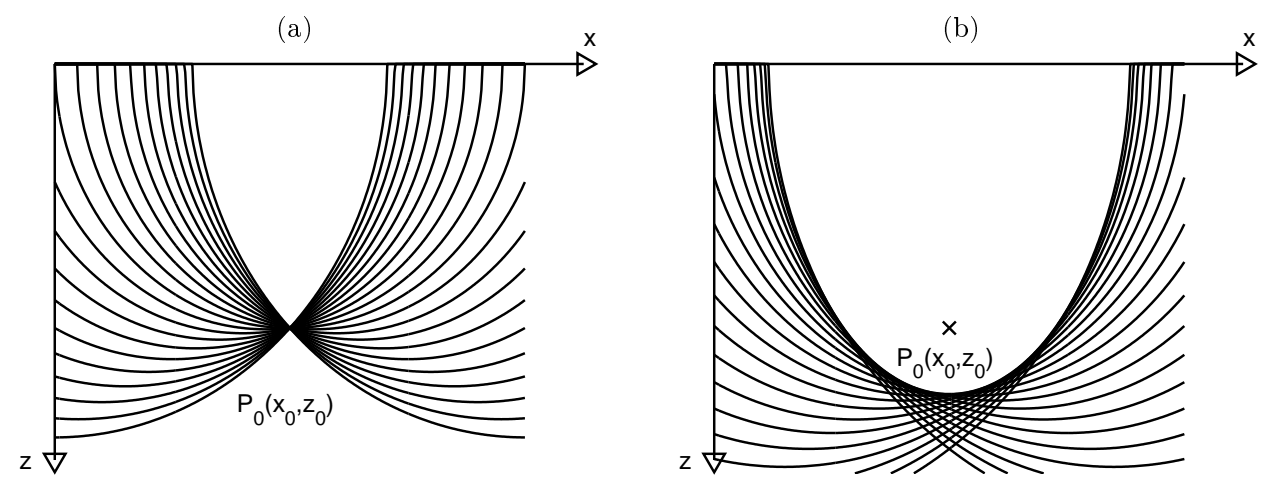

Figura 2: (a) Representação gráfica de como as isócronas se interceptam no ponto refletor $P_{0}\left(x_{0}, z_{0}\right)$. (b) Representação gráfica de como as isócronas não mais se interceptam no ponto refletor $P_{0}\left(x_{0}, z_{0}\right)$, aqui para uma velocidade $v>v_{0}$.

ponto $(\xi, 0)$ temos um mesmo tempo de propagação $t$ correspondente a este par fonte-receptor para todos os pontos $P(x, z)$ que satisfazem à equação

$$
z=\sqrt{\left(\frac{v_{0} t}{2}\right)^{2}-(x-\xi)^{2}} .
$$

Esta equação descreve uma semi-circunferência de raio $v_{0} t / 2$ que é chamada de isócrona, pois todos os pontos nesta curva possuem o mesmo tempo de propagação. Assim, cada valor de $\xi$ define um ponto $(\xi, 0)$ com seu respectivo $t(\xi)$ que gera uma nova isócrona. Todas essas isócronas passam por $P_{0}$ (Figura 2a). Esta construção é implicitamente realizada por uma migração com a velocidade $v_{0}$.

Porém, existe a possibilidade da velocidade de migração correta $v$ não ser $v_{0}$. Nesta situação, as isócronas do ponto $(\xi, 0)$ são descritas pela equação $(2.3) \operatorname{com} v_{0}$ substituído por $v$. A sua interseção não é mais o ponto $P_{0}\left(x_{0}, z_{0}\right)$ (ver Figura $2 \mathrm{~b}$ para o caso $v>v_{0}$ ) pois o raio da isócrona é proporcional à velocidade. $\mathrm{O}$ envelope do conjunto das isócronas representa a nova imagem do ponto refletor para a nova velocidade $v$. Observe que, para cada velocidade $v \neq v_{0}$, temos um novo envelope. Isto nos dá a idéia da propagação de uma onda imagem.

Agora temos que encontrar a equação que descreve estes envelopes pois assim temos a equação que descreve a posição da onda imagem. O envelope é descrito pela equação ([3])

$$
z=\left(v / v_{0}\right) \sqrt{z_{0}^{2}+\frac{\left(x-x_{0}\right)^{2}}{1-\left(v / v_{0}\right)^{2}}} .
$$

Calculando esta posição para vários valores de $v \neq v_{0}$, obtemos a propagação desta imagem.

O próximo passo consiste em estabelecer a relação entre esta parte cinemática da propagação da onda imagem e a correspondente equação diferencial parcial. 
Esta relação obedece aos mesmos princípios que a correspondente relação entre a cinemática da propagação de ondas acústicas e a equação da onda. No caso desta, a cinemática da propagação é descrita pela chamada equação iconal [1] . Correspondentemente, existe uma "equação iconal da onda imagem" que descreve a sua cinemática [3].

Para encontrarmos esta equação iconal para a onda imagem, substituímos a velocidade de migração $v$ da equação (2.4) por um iconal da onda imagem, $V(x, z)$, e calculamos as derivadas parciais desta equação modificada com respeito a $x$ e a $z$ [3]. Substituindo essas derivadas parciais novamente na equação (2.4) e realizando assim as eliminações de $x_{0}, z_{0}$ e $v_{0}$, obtemos a equação iconal da onda imagem

$$
V_{x}^{2}+V_{z}^{2}-\frac{V}{z} V_{z}=0
$$

que descreve a parte cinemática de uma solução da forma

$$
p(x, z, v)=p_{0}(x, z) f[v-V(x, z)]
$$

da equação da onda imagem (1.1) para o problema de remigração. Neste caso, desconsideramos uma função $F \equiv F\left(p_{x}, p_{z}, p_{v}, p, x, z, v\right)$ na equação (1.1) que não alteraria a equação iconal (2.5).

\section{Esquema de diferenças finitas}

Para utilização do método de diferenças finitas na equação (1.1), primeiramente, definimos uma malha de pontos $(x, z, v)$. Sejam $\Delta x, \Delta z$ e $\Delta v$ números positivos. A malha será os pontos $\left(x_{m}, z_{n}, v_{l}\right)=\left(x_{i}+m \Delta x, z_{i}+n \Delta z, v_{0}+l \Delta v\right)$ para quaisquer números inteiros $m, n$ e $l(m=1 \ldots M, n=1 \ldots N, l=1 \ldots L)$, onde $\left(x_{i}, z_{i}\right)$ representa o ponto inicial da malha espacial.

Assim, podemos utilizar uma aproximação com erro de truncamento local de quarta ordem para as derivadas segundas nas variáveis espaciais $x$ e $z$, de acordo com $[7,8]$. A segunda derivada em $x$ é aproximada por:

$$
\left(p_{x x}\right)_{m, n}^{l}=\frac{-p_{m+2, n}^{l}-p_{m-2, n}^{l}+16\left(p_{m+1, n}^{l}+p_{m-1, n}^{l}\right)-30 p_{m, n}^{l}}{12(\Delta x)^{2}}=\frac{\left(\delta_{x x} p\right)_{m, n}^{l}}{12(\Delta x)^{2}}
$$

e, em $z$, usamos a aproximação correspondente. Para a derivada mista utilizamos um esquema de diferenças finitas avançado em $v$ e em $z$, dado por

$$
\left(p_{z v}\right)_{m, n}^{l}=\frac{1}{\Delta v \Delta z}\left[p_{m, n+1}^{l+1}-p_{m, n}^{l+1}-p_{m, n+1}^{l}+p_{m, n}^{l}\right] .
$$

Como esta é uma aproximação com erro de truncamento local de primeira ordem nas variáveis $z$ e $v$, o esquema resultante também possui esta ordem nestas variáveis. A aproximação de quarta ordem para as derivadas segundas em $x$ e $z$ foi escolhida por fornecer resultados mais precisos em nossos experimentos numéricos do que a de segunda ordem $([5])$. 
Substituindo as aproximações (3.1) e (3.2) na equação (1.1) e isolando o termo $p_{m, n+1}^{l+1}$, encontramos

$$
p_{m, n+1}^{l+1}=p_{m, n}^{l+1}+p_{m, n+1}^{l}-p_{m, n}^{l}-\frac{z_{n} \Delta v \Delta z}{v_{l}}\left\{\frac{\left(\delta_{x x} p\right)_{m, n}^{l}}{12(\Delta x)^{2}}+\frac{\left(\delta_{z z} p\right)_{m, n}^{l}}{12(\Delta z)^{2}}\right\} .
$$

Para realizar a "propagação" da imagem utilizando este esquema, precisam-se de condições de contorno. Como o problema geofísico não fornece tais, introduzimos dois níveis anteriores nas direções $x$ e $z$ que supomos nulos.

No esquema (3.3), podemos observar que, para o cálculo do nível $l+1$, precisamse dos níveis $l+1$ e $l$. Assim, este é um esquema implícito. Porém, se tratássemos dele na implementação como tal, teríamos que resolver um sistema linear em cada passo da velocidade. Lembrando que estamos trabalhando com problemas de grande porte, onde este procedimento não seria viável, optamos por tratar do esquema (3.3) explicitamente, introduzindo uma condição de contorno adicional em $z$ no nível $l+1$, também nula, i.e., para o cálculo de $p_{m, 2}^{l+1}$ usa-se $p_{m, 1}^{l+1}=0$.

Observa-se que o esquema (3.3) pode ser implementado também na sua forma reversa em $z$, i.e.,

$$
p_{m, n}^{l+1}=p_{m, n+1}^{l+1}-p_{m, n+1}^{l}+p_{m, n}^{l}+\frac{z_{n} \Delta v \Delta z}{v_{l}}\left\{\frac{\left(\delta_{x x} p\right)_{m, n}^{l}}{12(\Delta x)^{2}}+\frac{\left(\delta_{z z} p\right)_{m, n}^{l}}{12(\Delta z)^{2}}\right\} .
$$

Nesta implementação, o cálculo de $p_{m, N-1}^{l+1}$ usa a condição de contorno adicional $p_{m, N}^{l+1}=0$.

\section{Análise do esquema numérico}

Um esquema de diferenças finitas pode ser utilizado para a determinação de uma solução aproximada de uma equação diferencial parcial, se a sua solução converge para a solução exata. Pelo Teorema de Eqüivalência de Lax ([7, 8]), um esquema é convergente se for consistente com a equação diferencial e estável. Analisamos, portanto, nesta seção, a consistência e estabilidade do esquema (3.3). É importante observar que esta análise vale também para o esquema (3.4), uma vez que este difere do (3.3) somente na sua forma de implementação.

\subsection{Consistência}

Um esquema de diferenças finitas é consistente com a correspondente equação diferencial parcial, se para qualquer função suave $\phi(x, z, v)$, a diferença entre o operador diferencial, $\mathcal{D}$, e o operador de discretização, $\mathcal{D}_{\Delta x, \Delta z}^{\Delta v}$, aplicada a $\phi$ tende a zero quando $\Delta v, \Delta x$ e $\Delta z$ tendem a zero $([7])$, ou seja,

$$
\mathcal{D} \phi-\mathcal{D}_{\Delta x, \Delta z}^{\Delta v} \phi \rightarrow 0, \quad \text { quando } \quad \Delta v, \Delta x, \Delta z \rightarrow 0 .
$$


Aplicando este conceito à equação (1.1), obtemos

$$
\begin{aligned}
\mathcal{D} \phi-\mathcal{D}_{\Delta x, \Delta z}^{\Delta v} \phi= & \phi_{x x}+\phi_{z z}+\left(v_{l} / z_{n}\right) \phi_{v z}-\left[\phi_{x x}+O\left(\Delta x^{4}\right)+\phi_{z z}+O\left(\Delta z^{4}\right)\right. \\
& \left.+\left(v_{l} / z_{n}\right)\left(\phi_{v z}+O(\Delta z)+O(\Delta v)\right)\right]
\end{aligned}
$$

$\operatorname{com} v_{l}=v_{0}+l \Delta v$ e $z_{n}=z_{i}+n \Delta z$. Assim,

$$
\mathcal{D} \phi-\mathcal{D}_{\Delta x, \Delta z}^{\Delta v} \phi=O\left(\Delta x^{4}\right)+O(\Delta z)+O(\Delta v) .
$$

Logo,

$$
\mathcal{D} \phi-\mathcal{D}_{\Delta x, \Delta z}^{\Delta v} \phi \rightarrow 0, \quad \text { quando } \quad \Delta v, \Delta x, \Delta z \rightarrow 0
$$

i.e., o esquema é incondicionalmente consistente com a equação diferencial (1.1).

\subsection{Estabilidade}

Para verificar a estabilidade do esquema de diferenças finitas, equação (3.3), aplicamos o critério de "von Neumann" ([7, 8]), i.e., substituímos na equação discretizada (3.3) a seguinte expressão

$$
p_{m, n}^{l}=\xi^{l} e^{i m k_{x} \Delta x} e^{i n k_{z} \Delta z} .
$$

Quando substituímos a expressão acima na equação (3.3) e dividimos esta pela equação (4.3), chegamos a

$$
\begin{aligned}
\xi=\frac{1}{\left(e^{i k_{z} \Delta z}-1\right)}\{ & \frac{16 z}{12 v} \frac{\Delta v \Delta z}{(\Delta x)^{2}} \operatorname{sen}^{2} \frac{k_{x} \Delta x}{2}\left[3+\operatorname{sen}^{2} \frac{k_{x} \Delta x}{2}\right] \\
& \left.+\frac{16 z}{12 v} \frac{\Delta v \Delta z}{(\Delta z)^{2}} \operatorname{sen}^{2} \frac{k_{z} \Delta z}{2}\left[3+\operatorname{sen}^{2} \frac{k_{z} \Delta z}{2}\right]\right\}+1,
\end{aligned}
$$

que, chamando a expressão entre chaves de $\lambda$, ainda pode ser reduzida à forma

$$
\xi=\frac{\lambda}{\left(e^{i k_{z} \Delta z}-1\right)}+1=\left(1-\frac{\lambda}{2}\right)-i\left(\frac{\lambda}{2} \frac{\cos \frac{k_{z} \Delta z}{2}}{\operatorname{sen} \frac{k_{z} \Delta z}{2}}\right) .
$$

Observamos que sen $\frac{k_{z} \Delta z}{2} \neq 0$, ou seja, $k_{z} \Delta z \neq 2 n \pi$, sendo $n$ um número natural.

Agora aplicamos o critério de estabilidade ([8]), ou seja, $|\xi| \leq 1$. No caso de $|\xi|=1$, notamos que $\lambda$ deve ser igual a zero o que implica que tanto sen $\frac{k_{x} \Delta x}{2}$ quanto sen $\frac{k_{z} \Delta z}{2}$ terão que ser iguais a zero. Porém, sen $\frac{k_{z} \Delta z}{2}=0$ contradiz a condição para a existência de $\xi$ (veja equação (4.4)). Logo, $\xi$ tem que satisfazer $|\xi|<1$. Assim, obtemos

$$
\lambda^{2}\left[\frac{1}{4 \operatorname{sen}^{2} \frac{k_{z} \Delta z}{2}}\right]-\lambda<0 .
$$


Como o coeficiente que multiplica $\lambda^{2}$ é sempre maior que zero, concluímos que $0<\lambda<4 \operatorname{sen}^{2} \frac{k_{z} \Delta z}{2}$. Logo,

$$
\begin{aligned}
0<\frac{z}{3 v} & \frac{\Delta v \Delta z}{(\Delta x)^{2}} \operatorname{sen}^{2} \frac{k_{x} \Delta x}{2}\left[3+\operatorname{sen}^{2} \frac{k_{x} \Delta x}{2}\right] \\
+ & \frac{z}{3 v} \frac{\Delta v \Delta z}{(\Delta z)^{2}} \operatorname{sen}^{2} \frac{k_{z} \Delta z}{2}\left[3+\operatorname{sen}^{2} \frac{k_{z} \Delta z}{2}\right]<\operatorname{sen}^{2} \frac{k_{z} \Delta z}{2} .
\end{aligned}
$$

Agora, vejamos o que acontece no caso de $\Delta x=\Delta z$ e $k_{x}=k_{z}$. Neste caso, $\operatorname{sen}^{2} \frac{k_{x} \Delta x}{2}=\operatorname{sen}^{2} \frac{k_{z} \Delta z}{2} \neq 0$. Dividindo a equação acima por este termo, obtemos então a condição

$$
0<\frac{4 z_{\max }}{3 v_{\min }} \frac{\Delta v}{\Delta z}+\frac{4 z_{\max }}{3 v_{\min }} \frac{\Delta v}{\Delta z}<1 \quad \Longrightarrow \quad \Delta v<\frac{3 v_{\min }}{8 z_{\max }} \Delta z,
$$

para que o esquema (3.3) seja estável para todos os valores de $v$ e $z$ envolvidos.

Observa-se, da equação (4.4), que

$$
\lim _{\operatorname{sen}\left(k_{z} \Delta z / 2\right) \rightarrow 0} \xi=1
$$

Assim, a condição (4.6) vale para todos os valores nuḿericos de $k_{z} \Delta z$, uma vez que não é possível fazer $k_{z} \Delta z$ assumir o valor de um múltiplo exato de $\pi$.

\section{Testes Numéricos}

Nesta seção mostramos um exemplo da aplicação do esquema de diferenças finitas (3.3) para a equação da onda imagem (1.1). Observa-se que a implementação teve que ser realizada de forma reversa na variável $z$, para evitar que o erro numérico cresça incontroladamente, mesmo que a condição de estabilidade (4.6) esteja satisfeita. Isto se deve à presença do fator $z$ que multiplica o termo da derivada mista. Este fator aumenta conforme $z$ cresce, ampliando assim o erro numérico acumulado dos passos anteriores. Quando o esquema é utilizado na direção de $z$ decrescente, o erro numérico é multiplicado por números cada vez menores e o problema é reduzido.

No exemplo mostrado abaixo, a região da remigração é entre -1500 m e 1500 m na direção $x$ e entre $10 \mathrm{~m}$ e $1600 \mathrm{~m}$ na direção $z$. A velocidade de migração da seção de entrada foi de $2000 \mathrm{~m} / \mathrm{s}$. Foram utilizados os valores de $\Delta x=\Delta z=10 \mathrm{~m}$ e $\Delta v=2 \mathrm{~m} / \mathrm{s}$, que é menor do que o valor de $\frac{3}{8} \frac{2000 \mathrm{~m} / \mathrm{s}}{1600 \mathrm{~m}} 10 \mathrm{~m}=\frac{75}{16} \mathrm{~m} / \mathrm{s} \approx 4.7 \mathrm{~m} / \mathrm{s}$, dado pela condição (4.6).

A Figura 3 mostra o modelo da terra a ser recuperado pelo processo da migração. Como uso na geofísica, o eixo $z$ aponta para baixo. O modelo é básico, consistindo de duas camadas homogêneas (representando dois tipos de rocha diferentes), separadas por uma interface horizontal na profundidade de $550 \mathrm{~m}$. A velocidade de propagação das ondas em cada camada é constante, sendo em cima da interface $3000 \mathrm{~m} / \mathrm{s}$ e abaixo dela $3500 \mathrm{~m} / \mathrm{s}$. Foi simulado numericamente um experimento sísmico de 301 pares de fontes e receptores coincidentes, localizados igualmente espaçados no eixo 


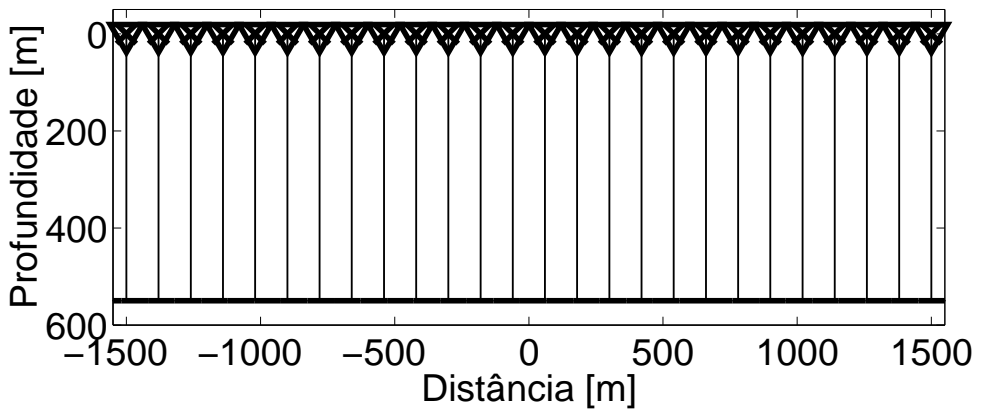

Figura 3: Modelo da terra utilizado para o teste da remigração.

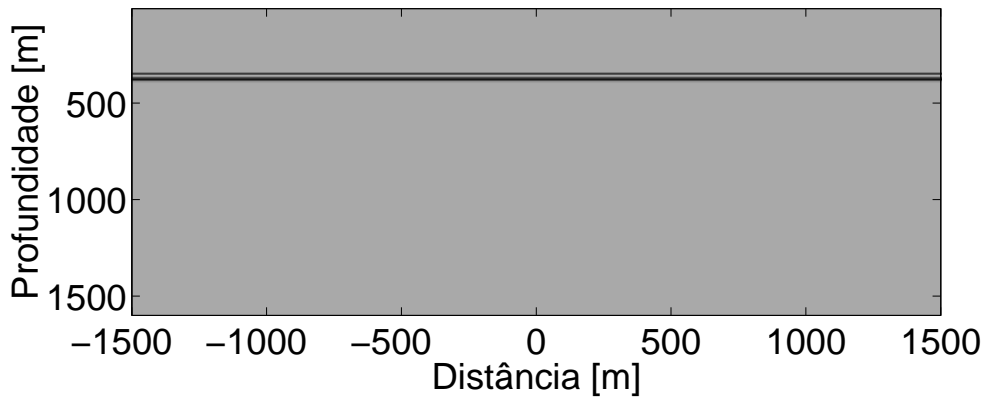

Figura 4: Seção sísmica migrada utilizando a velocidade de migração errada de $2000 \mathrm{~m} / \mathrm{s}$.

$x$ entre $-1500 \mathrm{~m}$ e $1500 \mathrm{~m}$. A Figura 3 mostra a correspondente família de raios refletidos do experimento.

Uma migração correta teria que ser aplicada, portanto, com a velocidade de migração de $3000 \mathrm{~m} / \mathrm{s}$ para fornecer uma imagem da interface na profundidade certa. Foi aplicada, porém, uma migração com a velocidade de migração errada de $2000 \mathrm{~m} / \mathrm{s}$, que fornece a imagem do refletor na profundidade errada, conforme mostra a Figura 4.

O objetivo do processo da remigração é a correção da localização desta imagem de acordo com uma nova velocidade de migração. A Figura 5(a,b,c) mostra a "propagação" da imagem do refletor para os valores de velocidade de migração de $2300 \mathrm{~m} / \mathrm{s}, 2700 \mathrm{~m} / \mathrm{s}$ e $3000 \mathrm{~m} / \mathrm{s}$, determinados pelo esquema de diferenças finitas (3.3). Observa-se na Figura 5(c) que corresponde à velocidade de migração correta, que a imagem do refletor se encontra na profundidade certa. O forte estiramento do pulso na remigração se deve à dispersão que é causada por um lado pelo esquema numérico e por outro lado pela própria equação da onda imagem (1.1).

Em testes numéricos adicionais com malhas espaciais mais refinadas $(\Delta x=\Delta z$ de $5 \mathrm{~m}$ e de $2.5 \mathrm{~m}$ ), o estiramento se reduziu visivelmente. No entanto, optamos 
(a)
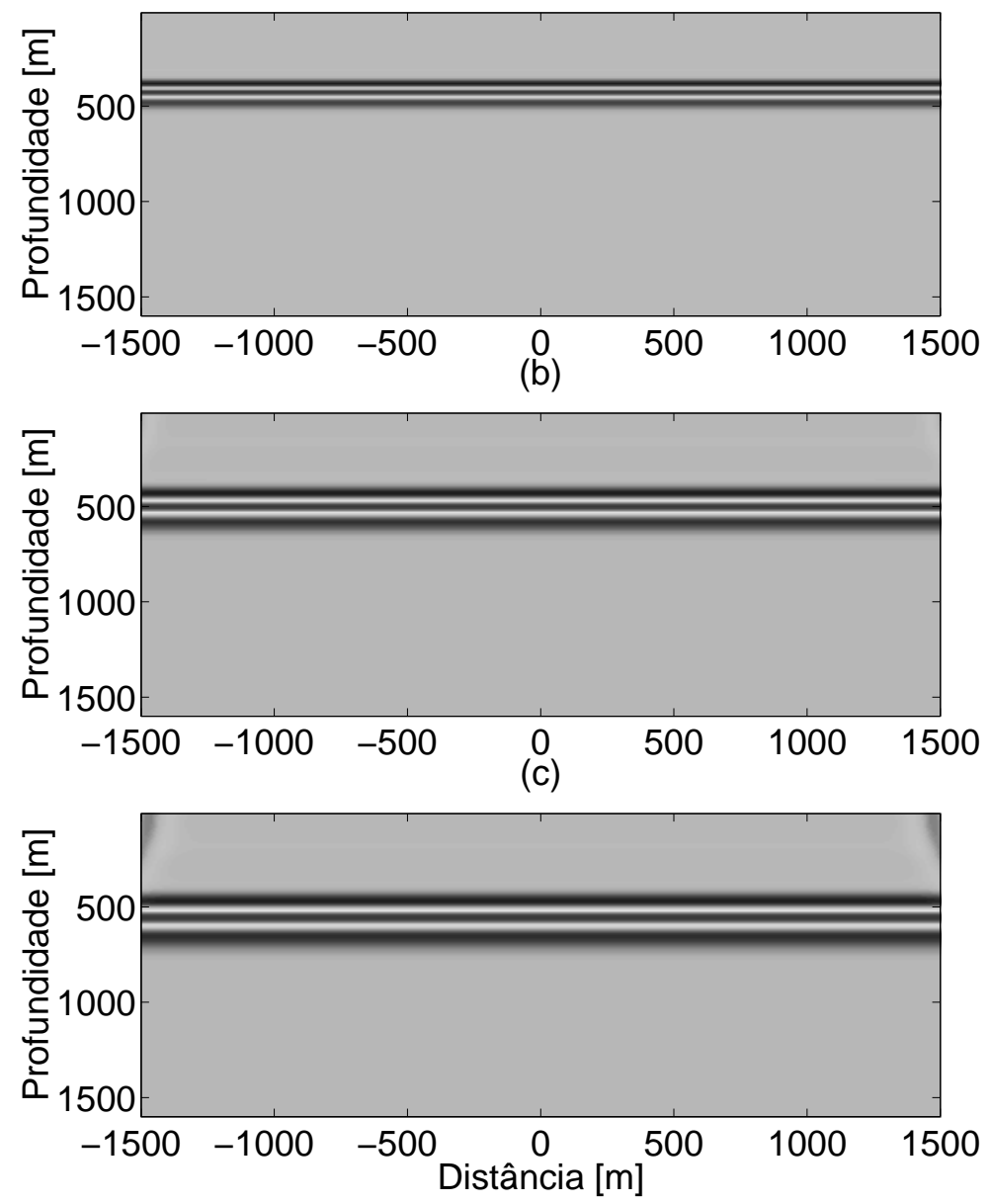

Figura 5: Seções sísmicas remigradas utilizando a equação da onda imagem, referentes às velocidades de migração: (a) $2300 \mathrm{~m} / \mathrm{s}$, (b) $2700 \mathrm{~m} / \mathrm{s}$, (c) $3000 \mathrm{~m} / \mathrm{s}$.

por incluir neste trabalho figuras obtidas com $\Delta x=\Delta z=10 \mathrm{~m}$, porque se trata do menor espaçamento usado na prática em métodos sísmicos. Assim, mostramos que é possível obter uma imagem estável usando uma malha convencional.

\section{Conclusões}

Neste trabalho, estudamos a equação diferencial parcial de segunda ordem conhecida como equação da onda imagem para remigração ([3]). Para esta equação, elaboramos um esquema de diferenças finitas de quarta ordem nas derivadas espa- 
ciais de segunda ordem e avançado nas duas variáveis da derivada mista. Para o esquema resultante, derivamos as condições de consistência e estabilidade. Testes numéricos mostram que o esquema, mesmo que estas condições estejam satisfeitas, pode apresentar erros numéricos muito grandes. Foi somente possível eliminar estes erros mediante a implementação reversa na variável que multiplica a derivada mista.

A propagação de uma imagem em função da velocidade de migração é muito desejável na sísmica, uma vez que a velocidade de migração correta geralmente não é conhecida e migrações com várias velocidades se tornam necessárias. Por meio da nossa implementação, mostramos que é possível determinar imagens do subsolo correspondentes a diferentes velocidades de migração utilizando a equação da onda imagem ao invés de múltiplas migrações.

\section{Agradecimentos}

Agradecemos à FAPESP, ao CNPq e aos patrocinadores do consórcio WIT pelo suporte financeiro.

Abstract. The image wave equation for remigration is a second-order partial differential equation that is similar to the acoustic wave equation. In this work, we determine the consistency and stability conditions for a finite difference scheme for this equation. In numerical tests can be observed that, in addition to the stability conditions, the form of implementation is essential to obtain acceptable numerical errors.

\section{Referências}

[1] N. Bleistein, "Mathematical Methods For Wave Phenomena", Academic Press, Orlando, Florida, 1984.

[2] S.B. Fomel, Method of velocity continuation in the problem of seismic time migration, Russian Geology and Geophysics, 35, No. 5 (1994), 100-111.

[3] P. Hubral, M. Tygel e J. Schleicher, Seismic image waves, Geophys, J. Int., 125 (1996), 431-442.

[4] M.S. Jaya, "Imaging reflection seismic data using the method of velocity continuation", Tese de Doutorado, Universidade de Karlsruhe, Alemanha, 1997.

[5] A. Novais e J. Schleicher, Propagação de ondas imagem por diferenças finitas, em "Resumos de XXIV CNMAC", p.577, 2001.

[6] R.H. Stolt e A.K. Benson, "Seismic Migration - Theory and Practice", Volume 5 da série Handbook of Geophysical Exploration, Geophysical Press, 1986.

[7] J.C. Strikwerda, "Finite Difference Schemes And Partial Differential Equations", Wadsworth \& Brooks, California, 1989.

[8] J.W. Thomas, "Numerical Partial Differential Equations", Springer-Verlag, New York, 1995. 